\title{
Topics in Computational Materials Science
}


This page is intentionally left blank 


\section{Topics in}

\section{Computational}

Materials

\section{Science}

Edited by

C Y Fong

University of California

Davis, California 


\section{Published by}

World Scientific Publishing Co. Pte. Ltd.

P O Box 128, Farrer Road, Singapore 912805

USA office: Suite 1B, 1060 Main Street, River Edge, NJ 07661

UK office: 57 Shelton Street, Covent Garden, London WC2H 9HE

\section{Library of Congress Cataloging-in-Publication Data}

Fong, C. Y. (Ching-yao)

Topics in computational materials science / C. Y. Wong.

p. $\mathrm{cm}$.

Includes bibliographical references and index.

ISBN 9810231490

1. Photonics -- materials -- Mathematical models. 2. Materials

science -- Mathematics. 3. Semiconductors -- Mathematical models.

I. Title.

TA1522.F66 1997

620.1'1297--dc21

$97-40545$

CIP

\section{British Library Cataloguing-in-Publication Data}

A catalogue record for this book is available from the British Library.

Copyright $\odot 1998$ by World Scientific Publishing Co. Pte. Ltd.

All rights reserved. This book, or parts thereof, may not be reproduced in any form or by any means, electronic or mechanical, including photocopying, recording or any information storage and retrieval system now known or to be invented, without written permission from the Publisher.

For photocopying of material in this volume, please pay a copying fee through the Copyright Clearance Center, Inc., 222 Rosewood Drive, Danvers, MA 01923, USA. In this case permission to photocopy is not required from the publisher.

This book is printed on acid-free paper.

Printed in Singapore by Uto-Print 


\section{FOREWORD}

The demands of understanding new experimental results for various types of growth, characterization of fascinating electronic devices and of striving effective guidelines for novel samples challenge computational materials science to make significant contributions. Among all the contributions in the past several years, I subjectively chose the following areas which are of current interests and are important for further advances:

(1) Development of efficient computational schemes for first-principles and realistic simulations of surfaces.

(2) The searching for ways to abate computational intensiveness in accurately determining the energy gap for semiconducting samples.

(3) Investigation of microscopic properties of photonic devices.

(4) Parametrization of interactions for large scale electronic calculations involving transition metal elements.

(5) Modeling, by many-body Hamiltonians or phenomenological potentials, for Monte Carlo or molecular dynamic simulations.

This book concerns specific topics in the above five areas. These topics are both crucial to the development of future computational materials science and for providing better microscopic understanding of materials used to making innovative electronic devices. I am fortunate to have had the privilege of inviting prominent authors who are authorities in their respective areas to contribute chapters for reviewing the current status and delineating directions for future research.

The $\mathrm{N}$-scaling scheme and parallel computing techniques are the principal elements for improving computational efficiency in realistic modeling involving a large number of atoms based on first-principles. They will be discussed in Chapter One by Dr. E. B. Stechel and in Chapter Two by Drs. J. S. Nelson and S. J. Plimpton. Professor S. G. Louie presents in Chapter Three the GW method for improving the semiconductor energy gaps over the results obtained by using the local density approximation of density functional theory; this improvement will provide meaningful comparisons with experimental data in the characterization processes. Since photonic devices are potential candidates for future device applications, photonic band calculations will be explained in Chapter Four by Drs. R. Biswas, M. M. Sigalas, C. M. Soukoulis, 
and Professor K. M. Ho. We realize that there are vast amount of experimental results to be understood on transition metal surfaces and defects involving transition metal elements. In Chapter Five, the tight-binding method for calculating electronic properties of large systems involving transition metal elements will be described by Drs. M. Mehl and D. Papaconstantopoulos. The modeling approaches are state-of-the-art alternatives to the first-principles simulated annealing method of Car and Parrinello for treating alloy problems and surface morphologies. The crucial ingredient for the success of these approaches is the determination of realistic interaction parameters. For alloy problems, Dr. Z. W. Lu and Professor B. M. Klein contribute Chapter Six to demonstrate how to study alloy properties by the Ising model using inputs determined from first-principles calculations. Professor E. Kaxiras enunciates, in Chapter Seven, computational methods for investigating epitaxial growth of semiconducting surfaces. Dr. M. Fallis and I contribute Chapter Eight on modeling close-packed metallic surfaces using the so-called bond saturation model. The parameters characterizing the model are determined from the embedded atom method and are used in Monte Carlo simulations of morphologies of adatoms in homoepitaxial growth. As several authors emphasize, it is hoped that this book will not only benefit researchers in these areas but will also serve as a starting point for scientists to devise new and more powerful methods.

I would like to thank A. Lavallee, N. Muniz, M. Hannon, J. E. Pask and Dr. M. Fallis for their word processing skills. Also, I thank A. Lavallee, N. Muniz, and J. L. Fong for the design of the book cover. Professor R. V. Reid and Charles Consorte's comments are greatly appreciated. Finally, I would like to thank Dr. K. K. Phua of the World Science Publishing Co. Ltd. for his invitation to edit this book. 


\section{TABLE OF CONTENTS}

Foreword

CHAPTER I. Advances in Algorithmic Development in the Electronic Structure of Large Systems

E. B. Stechel

1 Introduction

1.1 Motivation

1.2 Organization

2 Background and mathematical constructs

2.1 The variational principle

2.2 Density functional theory

2.3 The Harris functional, self-consistency and a variational principle

2.4 Representation theory

2.4.1 Initial setup

2.4.2 Primitive representation

2.4.3 Solution

2.4.4 Non-orthogonal algebra

2.5 n-representabiltity and the Green's function

2.6 The density matrix representation and idempotency 27

2.7 N-representability and invariant subspaces $\quad 29$

2.7.1 Generalized invariant subspace $\quad 35$

3 Linear scaling algorithms $\quad 37$

$\begin{array}{lll}3.1 \text { Green's functions } & 38\end{array}$

3.1.1 Divide and conquer $\quad 38$

3.1.2 Moment methods $\quad 40$

3.1.2.1 Lanczos algorithm $\quad 41$

3.1.2.2 Random vectors $\quad 42$

3.1.2.3 Chebyshev polynomials $\quad 43$

3.2 Density matrix algorithm $\quad 44$

3.3 Orbital based algorithms $\quad 46$

3.3.1 Unconstrained minimization 46

3.3.2 Non-orthogonal localized orbitals $\quad 47$

$\begin{array}{ll}3.3 .3 \text { Variants } & 49\end{array}$

4 Distorted grid 49

5 Summary 52 
Acknowledgments

Appendix A - The exchange-correlation potential in GGA 54

References

CHAPTER II. Plane Wave Pseudopotential Electronic Structure Calculations on Parallel Supercomputers

J. S. Nelson and S. J. Plimpton

1 Introduction

2 Evaluation of $\mathrm{HY} \quad 68$

3 Parallel issues

4 Benchmark timings $\quad 82$

5 Summary $\quad 84$

Acknowledgments $\quad 84$

References $\quad 84$

Appendix: Illustrative fortran subroutines 88

CHAPTER III. First-Principles Theory of Electron Excitation Energies in Solids, Surfaces, and Defects Steven G. Louie

1 Introduction 96

2 Theoretical concepts and methods 98

2.1 Quasiparticle excitations solids 98

2.2 Green's function and the electron self energy operator $\quad 100$

2.3 Calculation of self energies and the GW approximation 104

3 Some illustrative results 110

3.1 Bulk materials 110

$\begin{array}{ll}3.2 \text { Surfaces and interfaces } & 119\end{array}$

3.3 Materials under pressure and defects in solids 128

4 A Mixed-space imaginary time formulation 134

5 Summary and conclusion 136

$\begin{array}{ll}\text { Acknowledgments } & 137\end{array}$

$\begin{array}{ll}\text { References } & 137\end{array}$ 


\section{CHAPTER IV. Photonic Band Structure}

R. Biswas, M. M. Sigalas, C. M. Soukoulis, and $\mathrm{K} . \mathrm{M} . \mathrm{Ho}$

1 Introduction

2 Photonic band structure

3 Transfer matrix method (TMM)

4 Finite difference time domain (FDTD) method 162

Acknowledgments

References

\section{CHAPTER V. Tight-Binding Parametrization of First-Principles Results \\ Michael J. Mehl and Dimitrios A. Papaconstantopoulos}

1 Introduction

2 Tight-binding fundamentals

3 Slater-Koster parametrization of band structures

3.1 Monatomic metals

3.2 Semiconductors

3.3 The universal parameters of Harrison

4 Tight-binding total energy

5 Slater-Koster method for compounds

6 A new tight-binding method

6.1 A brief discussion of density functional theory

6.2 Development of a density functional theory-based Slater-Koster tight-binding parametrization

7 Examples of the method

7.1 Cubic transition metals - Palladium

7.2 A complicated transition metal - Manganese

$7.3 \mathrm{NbC}$ - a cubic carbide

$7.4 \mathrm{CoAl}$ - a cubic aluminide

7.5 $\mathrm{PdH}$ - a cubic hydride

8 Summary

Acknowledgments 
CHAPTER VI. First Principles Studies of Stability of Intermetallic Binary Alloys

Z. W. Lu and B. M. Klein

1 Introduction $\quad 214$

2 Method of calculation 218

2.1 Cluster-expanding lattice quantities 218

2.2 Applications of cluster expansion 224

3 Calculating the cluster expansion interaction energy 227

$\begin{array}{lll}3.1 \mathrm{Ag}-\text { Au system } & 227\end{array}$

3.2 Other binary systems 234

4 Low-temperature long-range order 236

5 Mixing energy of disordered alloys at finite temperature 239

6 High-temperature short-range order 241

7 Stability of superlattices and other complex structures 244

8 Effect of spin-polarization in alloy stabilities 249

9 Conclusion 256

Acknowledgments $\quad 256$

$\begin{array}{ll}\text { References } & 257\end{array}$

CHAPTER VII. Simulation of Semiconductor Growth Mechanisms in the Presence of Adsorbate Layers

Efthimios Kaxiras

1 Introduction 263

2 General considerations on growth processes 266

3 Quantum mechanical calculations 270

3.1 Overview of formalism for quantum mechanical
calculations

3.2 Total energy calculations of stable structures 276

3.3 Calculation of activation energies 282

4 Kinetic Monte Carlo simulations 287

5 Discussion and conclusions 296

Acknowledgments $\quad 301$

$\begin{array}{ll}\text { References } & 301\end{array}$ 
CHAPTER VIII. Bond Saturation Model: a Lattice Gas Approach for Studying Close Packed Metallic Surfaces

M. C. Fallis and C. Y. Fong

1 Introduction 306

1.1 Issues related to the simulation of metallic surface physics 306

1.2 Motivation for the bond saturation model 309

2 Bond Saturation Model (BSM) 311

2.1 Two dimensional BSM (2DBSM) 312

2.2 Three dimensional BSM (3DBSM) 319

2.3 Critical discussion 323

3 Application of the BSM to the Pt(111) system 325

3.1 Cluster energetics in the 2DBSM 326

3.2 Cluster energetics in the 3DBSM 334

3.3 High temperature island morphology 343

4 Conclusion $\quad 354$

Acknowledgments $\quad 354$

Appendix A - The embedded atom method 355

Appendix B - Quantum mechanical origin of coordination dependent bonding

References

Index 